\title{
New stable decagonal quasicrystal in the system Al-Ir-Os
}

\author{
S. Katrych ${ }^{\mathrm{a}}$, Th. Weber ${ }^{\mathrm{a}}$, M. Kobas ${ }^{\mathrm{b}}$, L. Massüger $^{\mathrm{a}}$, L. Palatinus ${ }^{\mathrm{c}, 1}$, \\ G. Chapuis ${ }^{\mathrm{c}}$, W. Steurer ${ }^{\mathrm{a}, *}$ \\ ${ }^{a}$ Laboratory of Crystallography, Department of Materials, ETH Zurich, Wolfgang-Pauli-Strasse 10, 8093 Zurich, Switzerland \\ ${ }^{\mathrm{b}}$ SLS Detector Group, Swiss Light Source, Paul-Scherrer-Institut, CH-5232 Villigen-PSI, Switzerland \\ ${ }^{\mathrm{c}}$ Laboratory of Crystallography, EPFL Lausanne, BSP-Dorigny, CH-1015 Lausanne, Switzerland
}

Received 7 March 2006; received in revised form 22 March 2006; accepted 22 March 2006

Available online 2 May 2006

\begin{abstract}
A stable decagonal quasicrystal with nominal composition $\mathrm{Al}_{73} \mathrm{Ir}_{14.5} \mathrm{Os}_{12.5}$ has been grown from the aluminum-rich melt. It has an incongruent melting temperature of $T_{\mathrm{m}}=1283^{\circ} \mathrm{C}$ and does not transform into a periodic phase within the maximum applied annealing time of 8 weeks at $1000^{\circ} \mathrm{C}$. The phase equlibria in the aluminum-rich part of the system Al-Ir-Os have been explored. The X-ray diffraction patterns can be indexed by five reciprocal basis vectors with lengths $a_{i}^{*}=0.2588(2) \AA^{-1}, i=1, \ldots, 4$, and $a_{5}^{*}=0.05945(3) \AA^{-1}$. This corresponds to a quasilattice parameter (i.e. the edge length of the related Penrose tiling) of 2.501(2) $\AA$ and a translation period along the 10-fold axis of 16.821(8) $\AA$. The 5D space group is $P 10_{5} \mathrm{mc}$. The structure of the decagonal phase has been solved by two different approaches, the 3D pseudo-approximant technique and the 5D charge-flipping method. It is closely related to those of decagonal Al-Os-Pd and Al-Ni-Ru and can be described as quasiperiodic packing of columnar clusters with $20.150 \AA$ diameter. The clusters consist of eight quasiperiodic layers with stacking sequence ....ABCDA' $\mathrm{B}^{\prime} \mathrm{C}^{\prime} \mathrm{D}^{\prime} \ldots$... where the prime (') denotes the layers generated by a $10_{5}$ operation.
\end{abstract}

(C) 2006 Elsevier B.V. All rights reserved.

Keywords: Quasicrystals; Intermetallics; Crystal structure; Metallography; X-ray diffraction

\section{Introduction}

There is some interest in intermetallic phases with high melting temperatures. In particular B2 phases (with CsCl-type structure) such as $\mathrm{NiAl}, \mathrm{RuAl}$ or IrAl show good high-temperature (HT) strength as well as reasonable toughness at ambient temperature. This motivated, for instance, the study of the phase diagram Al-Ir-Ru [1]. Although the substitution of ruthenium by osmium may also lead to interesting ternary phases with even higher melting temperature, nothing has been published yet on the ternary system $\mathrm{Al}-\mathrm{Ir}-\mathrm{Os}$. While the binary phase diagrams $\mathrm{Al}-\mathrm{Ir}$ and Ir-Os have been studied in detail [2], for the system $\mathrm{Al}-\mathrm{Os}$, only the structures of a few intermetallic phases have been determined yet. The binary systems Ir-Os and Ir-Ru each show a narrow miscibility gap and no intermetallic compounds. For the system Al-Ir the following intermetallics are known:

\footnotetext{
* Corresponding author. Tel.: +41 44632 6650; fax: +41 446321133 .

E-mail address: steurer@mat.ethz.ch (W. Steurer).

1 Permanent address: Institute of Physics, Academy of Sciences, Na Slovance 2, 18221 Prague, Czech Republic.
}

$\mathrm{Ir}_{2} \mathrm{Al}_{9}, \mathrm{Ir}_{13} \mathrm{Al}_{45}, \mathrm{Ir}_{4} \mathrm{Al}_{13}, \mathrm{Ir}_{9} \mathrm{Al}_{28}, \mathrm{IrAl}_{3}, \mathrm{IrAl}$ (see Refs. [3,4] and references therein). In the binary systems $\mathrm{Al}-\mathrm{Ru}$ and $\mathrm{Al}-\mathrm{Os}$ have been found: $\mathrm{RuAl}_{6}, \mathrm{Ru}_{4} \mathrm{Al}_{13}, \mathrm{Ru}_{2} \mathrm{Al}_{5}, \mathrm{RuAl}_{2}, \mathrm{Ru}_{2} \mathrm{Al}_{3}$, $\mathrm{RuAl}$ [5], and isotypic $\mathrm{Os}_{4} \mathrm{Al}_{13}, \mathrm{OsAl}_{2}, \mathrm{Os}_{2} \mathrm{Al}_{3}$, OsAl, respectively [2]. Therefore, there are good reasons to expect some similarity in the phase diagrams of the systems Al-Ir-Ru and Al-Ir-Os.

Metastable decagonal quasicrystals have been obtained by rapid solidification of $\mathrm{IrAl}_{4}, \mathrm{OsAl}_{5}$ and $\mathrm{RuAl}_{5}$ [6,7]. The translation period along the tenfold rotation axis is $\approx 16 \AA$ in the case of $\mathrm{d}-\mathrm{IrAl}_{4}$ and $\approx 4 \AA$ for $\mathrm{d}-\mathrm{Os} \mathrm{Al}_{5}$ and $\mathrm{d}-\mathrm{RuAl}_{5}$, respectively.

\section{Experimental}

The preparation of Al-Ir-Os samples is not straightforward due to the extremely different melting points of their components, $\mathrm{Al}: T_{\mathrm{m}}=660.4^{\circ} \mathrm{C}$, Ir: $T_{\mathrm{m}}=2410^{\circ} \mathrm{C}$, Os: $T_{\mathrm{m}}=3045^{\circ} \mathrm{C}$. The boiling point of aluminum is with $T_{\mathrm{b}}=2723^{\circ} \mathrm{C}$ somewhere in between the melting points of iridium and osmium. As a consequence, alloys obtained by melting just compacts of the element mixtures are porous, inhomogeneous and far from the starting composition due to aluminum evaporation. Therefore, we prepared our Al-Ir-Os samples, typically $\approx 1 \mathrm{~g}$ each, from Ir-Os master alloys and Al wire. At first, Ir-Os (Ir, Os: 99.9 wt.\%, Alfa Aesar) compacts were melted under Argon (99.998 wt.\%) in 
an arc furnace (MAM-1, Johanna Otto GmbH). Then, the Ir-Os melt is used to dissolve an $\mathrm{Al}$ wire (99.9 wt.\%, Alfa Aesar).

These prealloys were remelted and annealed in a high-vacuum resistance furnace (MOV 064, Pfeiffer Vakuum Anlagebau $\mathrm{GmbH}$ ) with subsequently cooling by jetting into the furnace chamber cold argon. The samples were characterized by standard metallographic techniques, powder X-Ray diffraction (PXRD) (Stoe, $\mathrm{Cu} \mathrm{K} \alpha_{1}$; Scintag, $\mathrm{Cu} \mathrm{K \alpha}$ ), and differential thermal analysis (DTA) (Perkin-Elmer DTA-7). The alloy compositions were determined by energy-dispersive X-Ray spectroscopy (EDX) at $15-30 \mathrm{kV}$ accelerating voltage on a "LEO 1530" analyzer using the VOYAGER software. Single-crystal Xray diffraction (SXRD) was carried out on a four-circle diffractometer equipped with CCD detector (Xcalibur PX, Oxford Diffraction, oscillation angle $1^{\circ}$, expo-

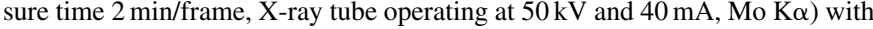
$65 \mathrm{~mm}$ sample-detector distance as well as on an imaging-plate-scanner (IPS) system (Marresearch 345, sample-to-image-plate distance $165 \mathrm{~mm}$, oscillation angle $0.5^{\circ}$, total range $180^{\circ}$, exposure time $20 \mathrm{~min} /$ frame, X-ray tube operating at $55 \mathrm{kV}$ and $25 \mathrm{~mA}$, graphite monochromatized $\mathrm{Ag} \mathrm{K} \alpha$ radiation).

\section{Results and discussion}

\subsection{Phase equilibria around the stability region of the decagonal phase}

In Fig. 1, a tentative concentration diagram of the ternary system Al-Ir-Os around the stability region of the decagonal phase is shown. As already mentioned above, there is no binary phase diagram available for the binary Al-Os system. Only data concerning the crystal structures of its compounds have been reported without any further information such as melting temperature or stability range [2]. Much more is known on the binary Al-Ir phase diagram [2], which we modified by adding the new phases $\operatorname{Ir}_{13} \mathrm{Al}_{45}$ and $\operatorname{Ir}_{9} \mathrm{Al}_{28}[3,4]$. We investigated the stability region of the decagonal phase in several isothermal sections. The powder diffractograms of the samples annealed for 3 weeks at $1000^{\circ} \mathrm{C}$ are depicted in Fig. 2.

The data of the PXRD analysis indicate the following phase fields at $1000^{\circ} \mathrm{C}$ : (Ir, Os) $\mathrm{Al}+(\mathrm{Ir}, \mathrm{Os}) \mathrm{Al}_{2}+(\mathrm{Ir}, \mathrm{Os}) \mathrm{Al}_{2.7}$, (Ir, Os) $\mathrm{Al}_{2}+(\mathrm{Ir}, \mathrm{Os}) \mathrm{Al}_{2.7},(\mathrm{Ir}, \mathrm{Os}) \mathrm{Al}_{2}+(\mathrm{Ir}, \mathrm{Os}) \mathrm{Al}_{2.7}+(\mathrm{Ir}, \mathrm{Os}) \mathrm{Al}_{3}$, $(\mathrm{Ir}, \mathrm{Os}) \mathrm{Al}_{2}+(\mathrm{Ir}, \mathrm{Os}) \mathrm{Al}_{3}, \mathrm{D}+(\mathrm{Ir}, \mathrm{Os})_{4} \mathrm{Al}_{13}+(\mathrm{Ir}, \mathrm{Os}) \mathrm{Al}_{3}, \mathrm{D}+(\mathrm{Ir}$,

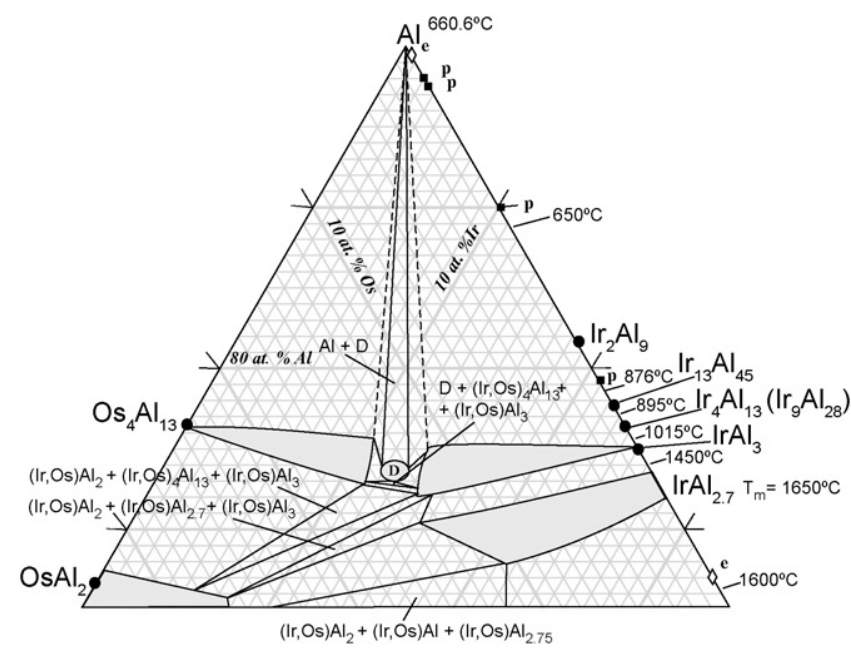

Fig. 1. Tentative solidus projection in the vicinity of the decagonal Al-Ir-Os phase field, D. e, p: mark eutectic and peritectic points, respectively. The corresponding temperatures are given as well. The shaded areas mark the single-phase fields.

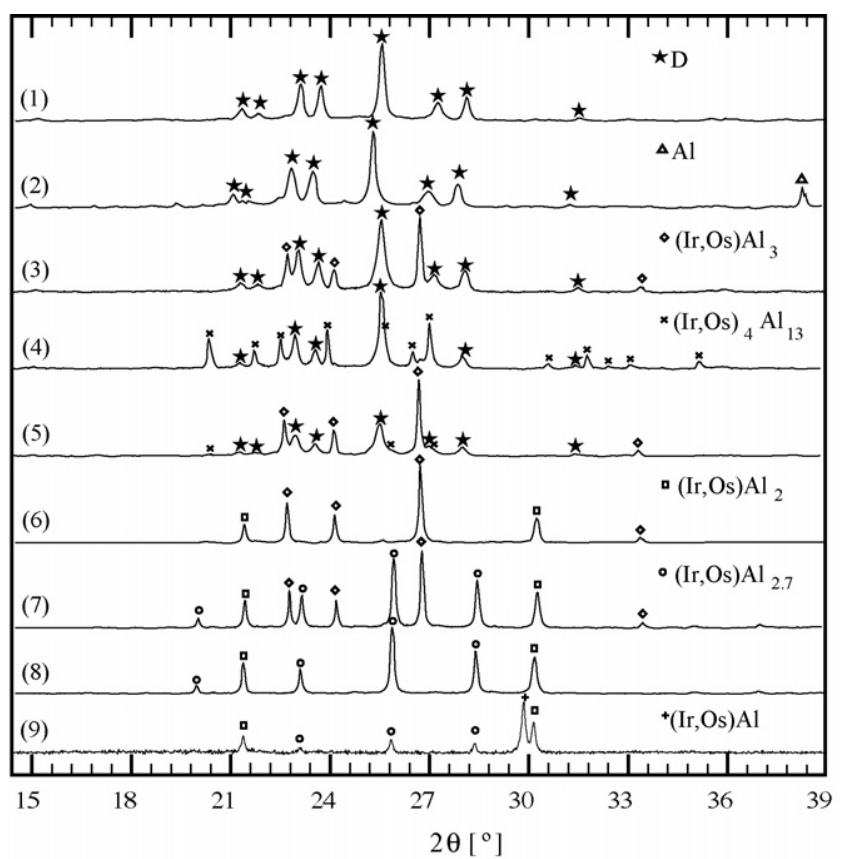

Fig. 2. X-ray powder diffraction patterns of Al-Ir-Os samples with compositions close to the stability region of the decagonal (D) phase. Samples 1 and 3-8 have been annealed for 3 weeks at $1000{ }^{\circ} \mathrm{C}$. Sample 2 was annealed 1 week at $800^{\circ} \mathrm{C}$ and sample 9 for $5 \mathrm{~h}$ at $1250^{\circ} \mathrm{C}$.

Os) ${ }_{4} \mathrm{Al}_{13}, \mathrm{D}+(\mathrm{Ir}, \mathrm{Os}) \mathrm{Al}_{3}, \mathrm{D}+\mathrm{Al}, \mathrm{D}$ (Fig. 1), where $\mathrm{D}$ is the new stable ternary decagonal phase. The two samples annealed at 1250 and $800^{\circ} \mathrm{C}$, respectively, show the same phase relationships. The DTA analysis of the samples from the phase regions: $(\mathrm{Ir}, \mathrm{Os}) \mathrm{Al}+(\mathrm{Ir}, \mathrm{Os}) \mathrm{Al}_{2}+(\mathrm{Ir}, \mathrm{Os}) \mathrm{Al}_{2.7},(\mathrm{Ir}, \mathrm{Os}) \mathrm{Al}_{2}+(\mathrm{Ir}, \mathrm{Os}) \mathrm{Al}_{2.7}$, (Ir, Os) $\mathrm{Al}_{2}+(\mathrm{Ir}, \mathrm{Os}) \mathrm{Al}_{2.7}+(\mathrm{Ir}, \mathrm{Os}) \mathrm{Al}_{3},(\mathrm{Ir}, \mathrm{Os}) \mathrm{Al}_{2}+(\mathrm{Ir}, \mathrm{Os}) \mathrm{Al}_{3}$ did not present any effects up to $1500^{\circ} \mathrm{C}$. The DTA curves of the samples from the other phase fields show two peaks at $\sim 1280$ and $\sim 1510^{\circ} \mathrm{C}$, respectively (Fig. 3).

Based on these results, the tentative solidus projection was constructed in the vicinity of the decagonal phase (Fig. 1). At $1250{ }^{\circ} \mathrm{C}, \mathrm{Al}_{2.7} \mathrm{Ir}$ dissolves $10.5-14$ at.\% Os. The lattice parameters of this cubic phase are $a=7.6876(3) \AA$ for the threephase sample with nominal composition $\mathrm{Al}_{66.5} \operatorname{Ir}_{21} \mathrm{Os}_{12.5}$ and $a=7.7219(1) \AA$ for the two-phase samples. The published lat-

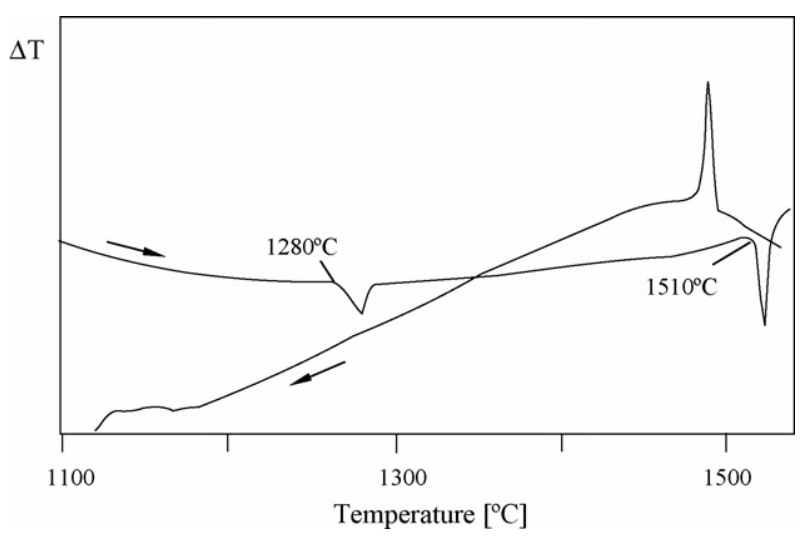

Fig. 3. DTA heating and cooling curves of a sample of the two-phase field $\mathrm{Al}_{13}(\mathrm{Ir}, \mathrm{Os})_{4}-\mathrm{D}$. 


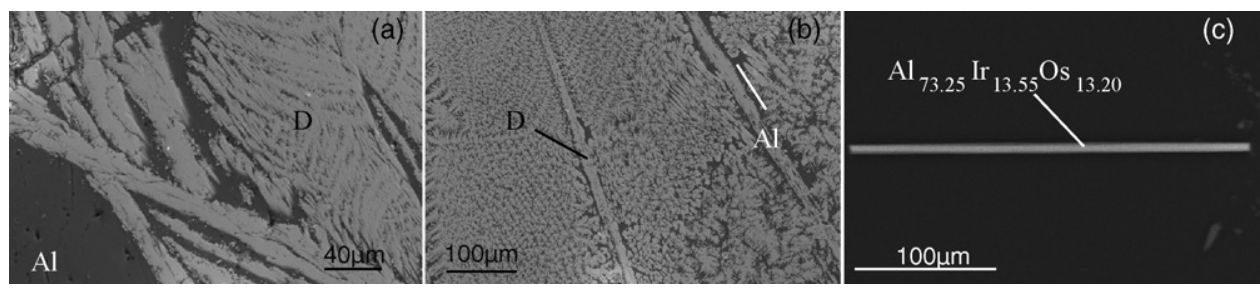

Fig. 4. SEM images of the $\mathrm{Al}-\mathrm{Ir}-\mathrm{Os}$ alloys (a) $\mathrm{Al}_{89.2} \mathrm{Ir}_{5.6} \mathrm{Os}_{5.2}$ and (b) $\mathrm{Al}_{83.9} \mathrm{Ir}_{9.1} \mathrm{Oss}_{7}$, annealed $168 \mathrm{~h}$ at $800{ }^{\circ} \mathrm{C}$. (c) $\approx 10 \mu \mathrm{m}$ thick needle-like single crystal of the d-phase with nominal composition $\mathrm{Al}_{73.25} \operatorname{Ir}_{13.55} \mathrm{Os}_{13.20}$, grown during a DTA cooling cycle $\left(5^{\circ} \mathrm{C} \mathrm{min}^{-1}\right)$.

tice parameter for the binary $\operatorname{IrAl}_{2.7}$ phase in the single-phase sample annealed at $600^{\circ} \mathrm{C}$ is $a=7.6789(3) \AA$ [8]. The solubility of $\mathrm{Ir}$ in $\mathrm{OsAl}_{2}$ at $1250^{\circ} \mathrm{C}$ amounts to about 8 at.\%. The lattice parameters of this tetragonal phase, derived from the three phase samples $\mathrm{OsAl}_{2}+(\mathrm{Ir}, \mathrm{Os}) \mathrm{Al}+(\mathrm{Ir}, \mathrm{Os}) \mathrm{Al}_{2.7}$ annealed at $1250{ }^{\circ} \mathrm{C}$, are in the range from $a=3.16485(9)$ to $3.16417(9) \AA$ and $c=8.2955(3)$ to $8.29530(3) \AA$ compared to $a=3.162 \AA$ and $c=8.302 \AA$ for the single-phase sample [9].

There is a large solubility of the third component in the binary phases. At $800^{\circ} \mathrm{C}$ between 10 and 11.5 at. $\%$ Ir can be dissolved in $\mathrm{Os}_{4} \mathrm{Al}_{13}$. The lattice parameters of a single crystal resulted to $a=7.543(1) \AA, b=4.2545(3) \AA$, $c=7.7533(6) \AA, \quad \beta=116.038(6)^{\circ}$ compared to $a=7.64 \AA$, $b=4.228 \AA, c=7.773 \AA, \beta=115.15^{\circ}$ for the Ir-free phase [10]. The solubility of Os in $\mathrm{IrAl}_{3}$ amounts to $11-13.5$ at.\% at $1200^{\circ} \mathrm{C}$. The lattice parameters obtained from single-crystal Xray diffraction data are $a=4.259(1) \AA, c=7.815(1) \AA, \gamma=120^{\circ}$ compared to $a=4.250 \AA, c=7.760 \AA, \gamma=120^{\circ}$ for pure $\mathrm{IrAl}_{3}$ [11]. The phase relationships in the regions $\mathrm{Os}_{4} \mathrm{Al}_{13}-\mathrm{Al}-\mathrm{D}$ and $\mathrm{IrAl}_{3}-\mathrm{Al}-\mathrm{D}$ are still not clear.

\subsection{Crystal preparation}

Single crystals of the decagonal phase (d-phase, in the previous chapter denoted $\mathrm{D}$ ) have been obtained from aluminumrich two-phase samples. Metallographic SEM images of these samples with compositions $\mathrm{Al}_{83.9} \mathrm{Ir}_{9.1} \mathrm{Os}_{7}$ and $\mathrm{Al}_{89.2} \mathrm{Ir}_{5.6} \mathrm{Os}_{5.2}$, which were annealed for $168 \mathrm{~h}$ at $800{ }^{\circ} \mathrm{C}$, show the decagonal phase in equilibrium with aluminum (Fig. 4). The DTA curves (Fig. 5) show the aluminum melting/solidification peaks and, at $\approx 1293{ }^{\circ} \mathrm{C}$, a peak related to the incongruent melting of the dphase (Fig. 4b). A further peak, due to the limited range of our DTA instrument observable only in the cooling cycle, marks the liquidus curve. During the DTA cooling cycle $\left(5 \mathrm{~K} \mathrm{~min}^{-1}\right)$ large needle-shaped crystals of the decagonal phase grew from the melt (Fig. 4c). The composition of these single crystals was determined by EDX to $\mathrm{Al}_{73} \mathrm{Ir}_{14.5} \mathrm{Os}_{12.5}$ and $\mathrm{Al}_{73.25} \mathrm{Ir}_{13.55} \mathrm{Os}_{13.2}$, respectively.

The PXRD patterns of the two-phase samples shown in Fig. $4 \mathrm{a}$ and $\mathrm{b}$ exhibit $\mathrm{Al}$ peaks beside those of the decagonal phase (Fig. 6). Neglecting the Al-peaks, the spectra show close resemblance to those of the (pseudo)decagonal phases in the systems Al-Pd-Me (Me: Fe, Ru, Os) [12,13].

\subsection{Single-crystal $X$-ray diffraction}

A single-crystal X-ray diffraction study of the new decagonal Al-Ir-Os phase confirms its quasiperiodicity and

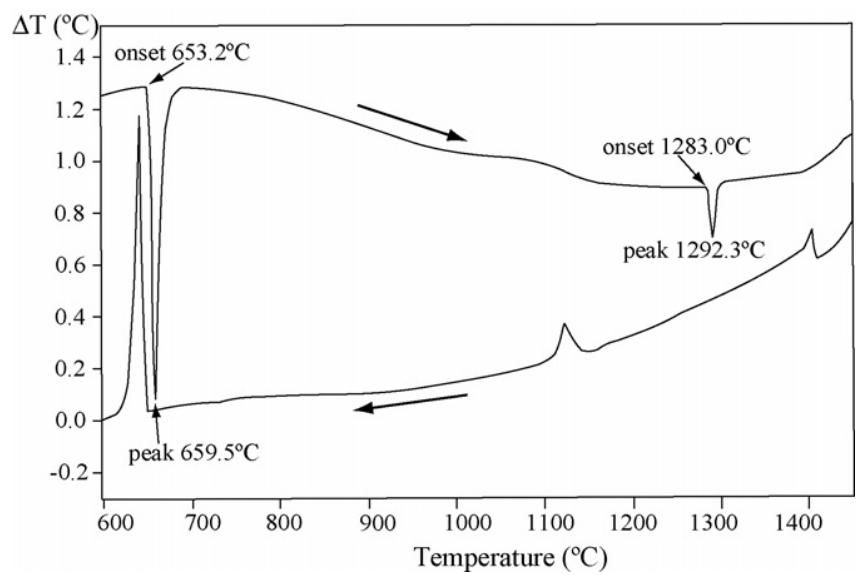

Fig. 5. DTA heating and cooling curves of the two-phase sample with composition $\mathrm{Al}_{83.9} \mathrm{Ir}_{9.1} \mathrm{Os}_{7}$ (Fig. $4 \mathrm{~b}$ ) annealed $168 \mathrm{~h}$ at $800^{\circ} \mathrm{C}$. The first peak results from aluminum melting, the second from the incongruent melting of the $\mathrm{d}$ phase. The peak at $1450{ }^{\circ} \mathrm{C}$ on the cooling curve indicates the liquidus curve. The heating/cooling rate was $10^{\circ} \mathrm{C} \mathrm{min}^{-1}$.

10 -fold symmetry, i.e. Laue group 10/mmm. Contrary to (pseudo)decagonal Al-Pd-Fe [13] and Al-Pd-Ru [13,14], decagonal Al-Ir-Os does not show any reflection splitting or symmetry breaking. All reflections can be indexed with quintuplets of integers with diffraction vectors $\mathbf{H}=\sum_{i=1}^{5} h_{i} \mathbf{a}_{i}^{*}$ on the basis $\mathbf{a}_{i}^{*}=a_{i}^{*}(\cos 2 \pi i / 5, \sin 2 \pi i / 5,0), i=1, \ldots, 4$, and $\mathbf{a}_{5}^{*}=a_{5}^{*}(0,0,1)$ (see Ref. [15]). With $a_{i}^{*}=0.2588(2) \AA^{-1}$, $i=1, \ldots, 4, a_{5}^{*}=0.05945(3) \AA^{-1}$ derived from IPS data, we obtain $a_{\mathrm{r}}=2 \tau / 5 a_{i}^{*}=2.501(2) \AA^{-1}$ for the quasilattice

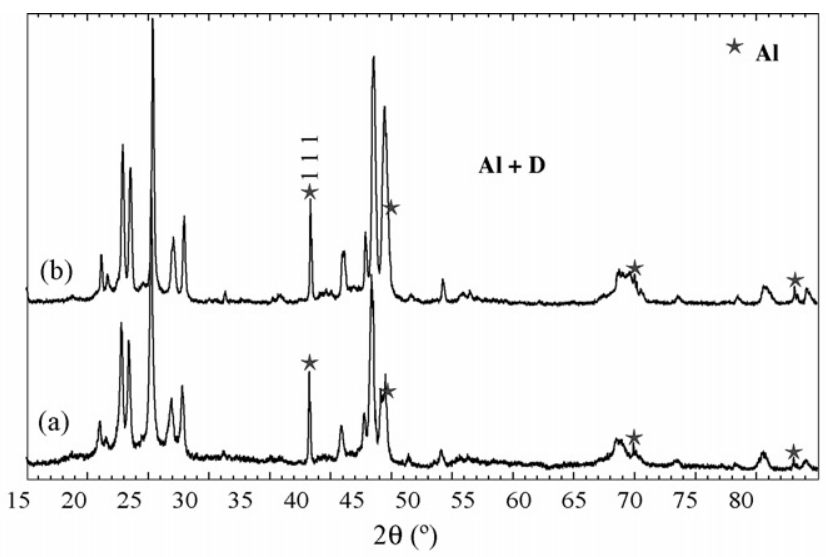

Fig. 6. Powder X-ray diffraction patterns of: (a) the $\mathrm{Al}_{89.2} \mathrm{Ir}_{5.6} \mathrm{Os}_{5.2}$ sample annealed $168 \mathrm{~h}$ at $800^{\circ} \mathrm{C}$; (b) the $\mathrm{Al}_{83.9} \mathrm{Ir}_{9.1} \mathrm{Os}_{7}$ sample annealed $168 \mathrm{~h}$ at $800^{\circ} \mathrm{C}$. Both samples show aluminum for the second phase (see Fig. 4a and b). 


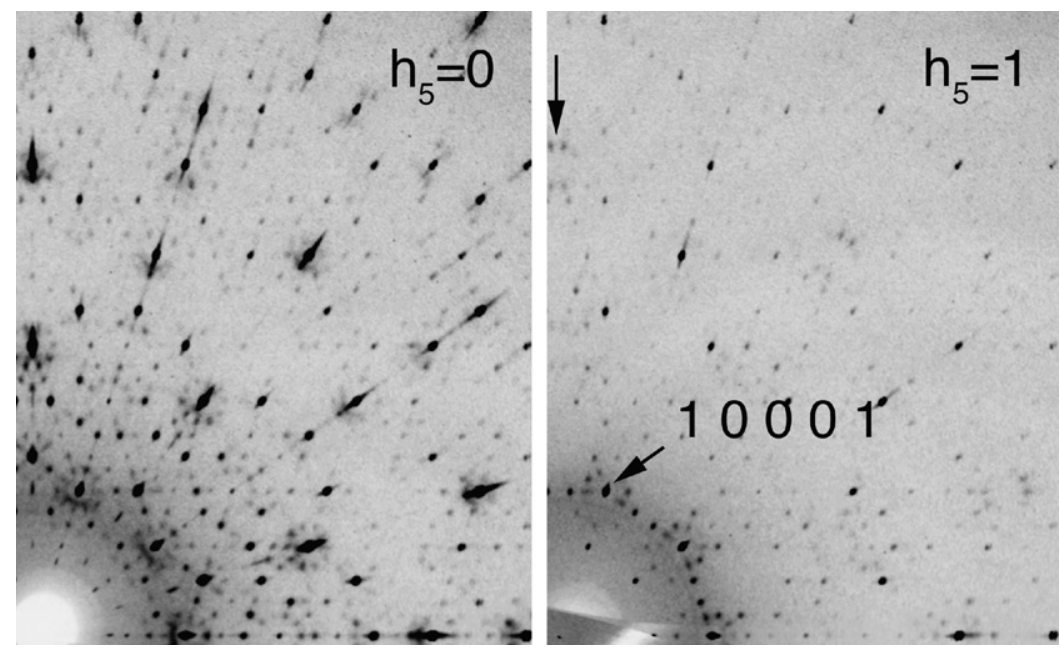

Fig. 7. Reciprocal space sections of decagonal Al-Ir-Os reconstructed from 360 image plate scanner frames (exposure time $20 \mathrm{~min} / \mathrm{frame}, \Delta \phi=0.5^{\circ}, \mathrm{Ag} \mathrm{K} \alpha$ radiation). The reciprocal space layers $h_{1} h_{2} h_{3} h_{4} h_{5}$ with $h_{5}=0$ and 1 are displayed. The 10001 reflection is marked in the right image as well as the direction of systematic extinctions $h_{1} h_{2} \bar{h}_{2} \bar{h}_{1} h_{5}$ (arrow pointing down), which corresponds to Fig. 8b.

parameter (edge length of the related Penrose tiling) and $c=1 / a_{5}^{*}=16.821(8) \AA$ for the translation period along the 10 -fold axis. For comparison, for isotypic decagonal Al-Os-Pd these values are $a_{i}^{*}=0.25650(3) \AA^{-1}, i=1, \ldots$, $4, \quad a_{5}^{*}=0.05970(3) \AA^{-1}, \quad a_{\mathrm{r}}=2 \tau / 5 a_{i}^{*}=2.5233(2) \AA \quad$ and $c=1 / a_{5}^{*}=16.750(3) \AA^{-1}[16]$. The difference in quasilattice parameters, $\quad a_{\mathrm{r}}^{\mathrm{Al}-\mathrm{Os}-\mathrm{Pd}}-a_{\mathrm{r}}^{\mathrm{Al}-\mathrm{Ir}-\mathrm{Os}}=2.523 \AA-2.501 \AA=$ $0.022 \AA$, corresponds to the difference in atomic radii of $\mathrm{Pd}$ and Ir, $1.376 \AA-1.357 \AA=0.019 \AA$. For the other isotypic decagonal phase, Al-Ni-Ru we obtained the larger reciprocal lattice parameters $a_{i}^{*}=0.26068(3) \AA^{-1}, i=1, \ldots, 4$, $a_{5}^{*}=0.060464(9) \AA^{-1}$, corresponding to $a_{\mathrm{r}}=2.4828(4) \AA$ and $c=16.539(3) \AA$ [17]. The smaller quasilattice parameter results from the much smaller atomic radius of $\mathrm{Ni}, 1.246 \AA$, compared to that of Ir and Os.

The reciprocal space layers $h_{1} h_{2} h_{3} h_{4} h_{5}$ with $h_{5}=0$ and 1 are shown in Fig. 7. The layers $h_{1} h_{2} h_{2} h_{1} h_{5}$ and $h_{1} h_{2} \bar{h}_{2} \bar{h}_{1} h_{5}$ perpendicular to them are depicted in Fig. 8. There are only a few weak violations of the systematic extinctions $h_{1} h_{2} \bar{h}_{2} \bar{h}_{1} h_{5}$ : $h_{5}=2 n+1$ indicating that the 5D space group $P 10_{5} / m m c$ or its non-centrosymmetric subgroup $P 10_{5} m c$ is not the strict but a reasonably good symmetry description. Pronounced ring-like diffuse scattering around (and not beneath) strong reflections, in particular in the sixth reciprocal-space layer, indicates some orientational disorder.

\subsection{D structure analysis by the pseudo-approximant method}

The goal of the pseudo-approximant method is to obtain quick and easily information on the local structure of a quasiperiodic structure. A quasicrystal and its rational $n / n^{\prime}-$ approximant are structurally closely related. In the higherdimensional approach, a $n / n^{\prime}$ - approximant results from a particular perpendicular-space shear of the parent hypercrystal. Quasiperiodic as well as approximant structures can be obtained by cutting the hypercrystal by the physical space. In case of the quasicrystal, the slope of the physical space corresponds to an irrational number, in case of a rational approximant to a rational number (for a detailed explanation see Refs. $[15,18]$ ).

In case of the pseudo-approximant method, not a really existing approximant is used, instead, the reciprocal space of the
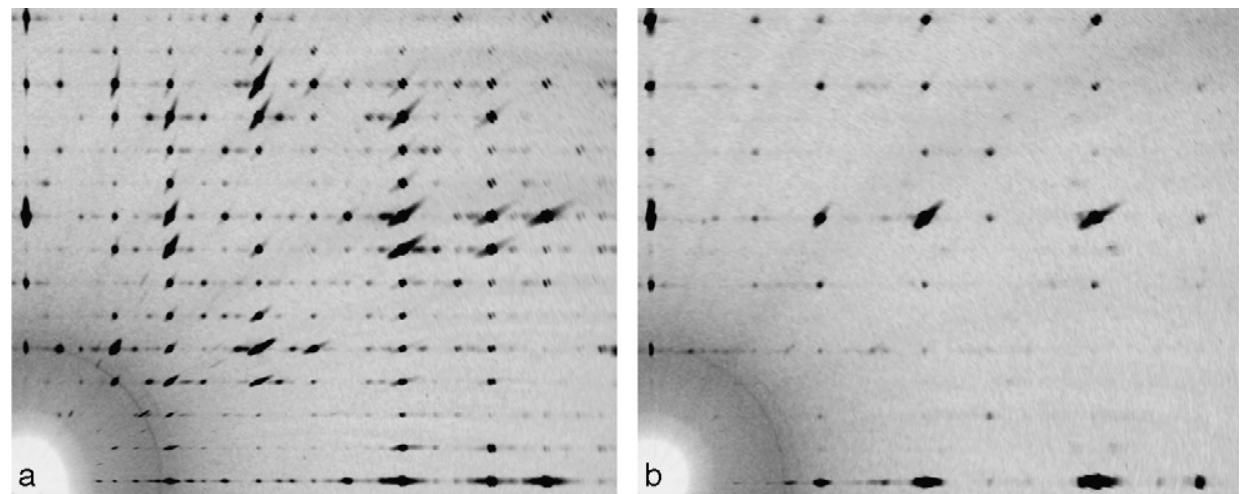

Fig. 8. Reciprocal space sections of decagonal Al-Ir-Os reconstructed from 360 image plate scanner frames (exposure time $20 \mathrm{~min} /$ frame, $\Delta \phi=0.5^{\circ}, \mathrm{Ag} \mathrm{K} \alpha$ radiation). The reciprocal space layers (a) $h_{1} h_{2} h_{2} h_{1} h_{5}$ and (b) $h_{1} h_{2} \bar{h}_{2} \bar{h}_{1} h_{5}$ are displayed. 
quasicrystal is transformed into that of the approximant. Then the structure of the approximant is solved by standard crystallographic methods and refined to get the phases of the experimental structure amplitudes. The Fourier transform of the structure factors obtained in this way give electron density maps, which show the characteristic building units (clusters). This works properly, however, only if the unit cell of the approximant is significantly larger than the cluster.

Consequently, in order to get a first idea about the local structure of d-Al-Ir-Os, its reciprocal space was transformed into that of the rational 3/5-approximant. The 3/5-approximant was chosen because such an approximant, with lattice parameters $a=23.889(3) \AA, b=32.802(3) \AA, c=16.692(1) \AA$, was experimentally observed in the system Al-Pd-Ru [14]. Furthermore, it is of a size, which can be easily handled by standard structure determination methods. The lattice parameters of the 3/5approximant can be calculated using the formula [18]:

$a_{1}^{\mathrm{app}}=\frac{2(3-\tau)}{5 a^{*}} \tau^{n+2}=a_{\mathrm{r}}(3-\tau) \tau^{n}$,

$a_{2}^{\mathrm{app}}=\frac{2 \sqrt{3-\tau}}{5 a^{*}} \tau^{n^{\prime}+1}=a_{\mathrm{r}} \sqrt{3-\tau} \tau^{n^{\prime}-1}$,

$a_{3}^{\mathrm{app}}=\frac{1}{a_{5}^{*}}$.
For $n=3$ and $n^{\prime}=5$ we obtain $a_{1}^{\text {app }}=23.901 \AA, a_{2}^{\text {app }}=32.896 \AA$, $a_{3}^{\mathrm{app}}=16.821 \AA$, and the indices result to

$h_{1}^{\mathrm{app}}=-5\left(h_{2}+h_{3}\right)+2\left(h_{1}+h_{4}\right)$,

$h_{2}^{\text {app }}=8\left(h_{1}-h_{4}\right)+5\left(h_{2}-h_{3}\right)$,

$h_{3}^{\mathrm{app}}=h_{5}$.

Since $n \bmod 3=\left(n^{\prime}+1\right) \bmod 3$, the $3 / 5$-approximant is $C$ centered orthorhombic. The quasicrystal data set contains 15354 reflections with $I>4 \sigma(I)\left(R_{\text {int }}=0.075 ; 4.17^{\circ} \leq \theta \leq 44.93^{\circ}\right.$; Mo $\mathrm{K} \alpha$ ), which were merged to 5191 unique reflections. Subsequently, this data set was transformed into that of the $3 / 5$ approximant. Its diffraction pattern shows close resemblance to those of $\operatorname{Ir}_{9} \mathrm{Al}_{28}$ and $\mathrm{Ir}_{13} \mathrm{Al}_{45}$ (Fig. 9). Consequently, these periodic phases can also be considered as crystalline approximants of the d-phase. This means, both the d-phase and the approximants are built from (at least in projection) similar structural subunits.

Its Laue group is the subgroup mmm of the Laue group of the d-phase, 10/mmm. Based on systematic extinctions, the space group can be either $C \mathrm{cmm}$ (No. 63) or one of its subgroups $C c 2 m$ (No. 40) and $C c m 2_{1}$ (No. 36), respectively. $C c 2 m$, however, is unlikely since it neither has a 21 parallel to the $10_{5}$-axis of the d-phase nor a $m$ perpendicular to $c$ as existing in the d-phase.
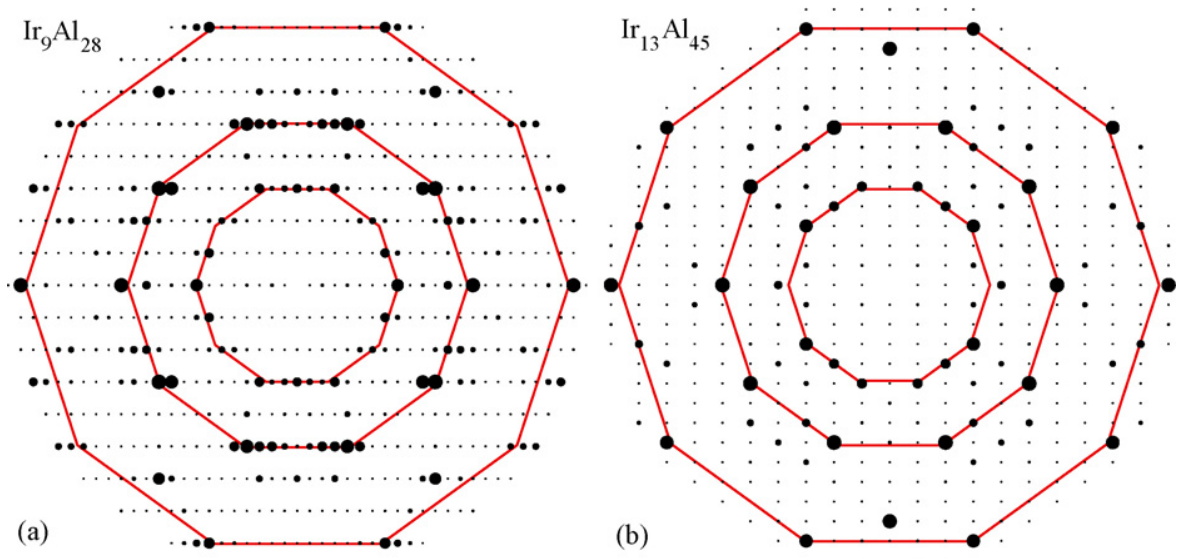

(c)

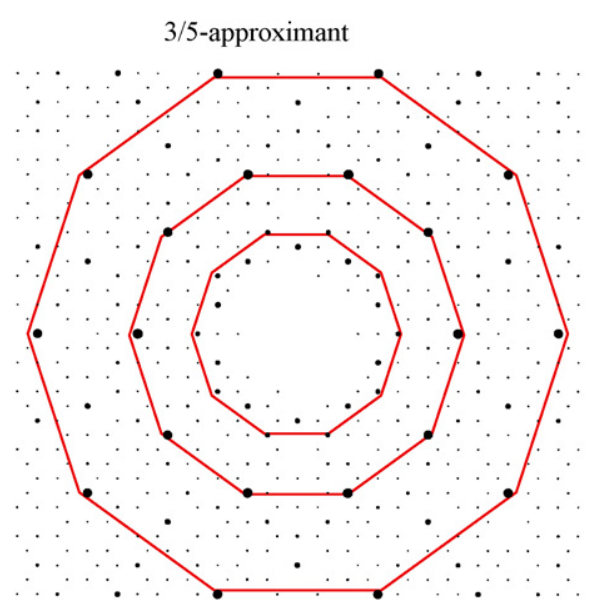

Fig. 9. Calculated $(0 k l)$ reciprocal space sections of (a) $\operatorname{Ir}_{9} \mathrm{Al}_{28}$ [4], (b) $\operatorname{Ir}_{13} \mathrm{Al}_{45}$ [3] in comparison with (c) the ( $h k 0$ ) section of the hypothetical 3/5-approximant. The pseudo-10-fold symmetry is indicated by decagons. 
The action of a 21 -axis on a columnar cluster with pentagonal symmetry is equivalent to that of a $10_{5}$-axis. Refinements in the two remaining space groups lead to similar results. Finally, Ccm $2_{1}$ (No. 36) was chosen because it yielded the lowest Rfactors and the least number of split positions. The structure was solved by direct methods [19] and subsequently refined to $R=0.125$ (5191 reflections with $I>4 \sigma(I), 894$ parameters; unit weights) [20].

The electron density maps of the four puckered layers are shown in Fig. 10. Aluminum atoms are barely visible. They have only about one sixth of the scattering power of Ir and Os atoms, which themselves cannot be distinguished by X-ray scattering. Furthermore, particularly Al atoms suffer from disorder, i.e. in the average structure their already low electron density is smeared over split positions. According to the edge length of the Penrose rhomb (marked in Fig. 10), the diameter of the unit cluster (structural repeat unit) results to $20.331 \AA$. As far as it can be compared, its structure shows close similarities to that of decagonal Al-Ni-Ru [17,21].

\subsection{D structure analysis by the charge-flipping method}

Charge flipping is a relatively new iterative method for $a b$ initio phasing of structure factors $[22,23]$. Soon after its publication it was shown that it is applicable also for the solution of incommensurately modulated structures (IMS) using a superspace formalism [24]. In the $(3+1)$-dimensional superspace description of IMS, the hyperatoms form continuous strings. The
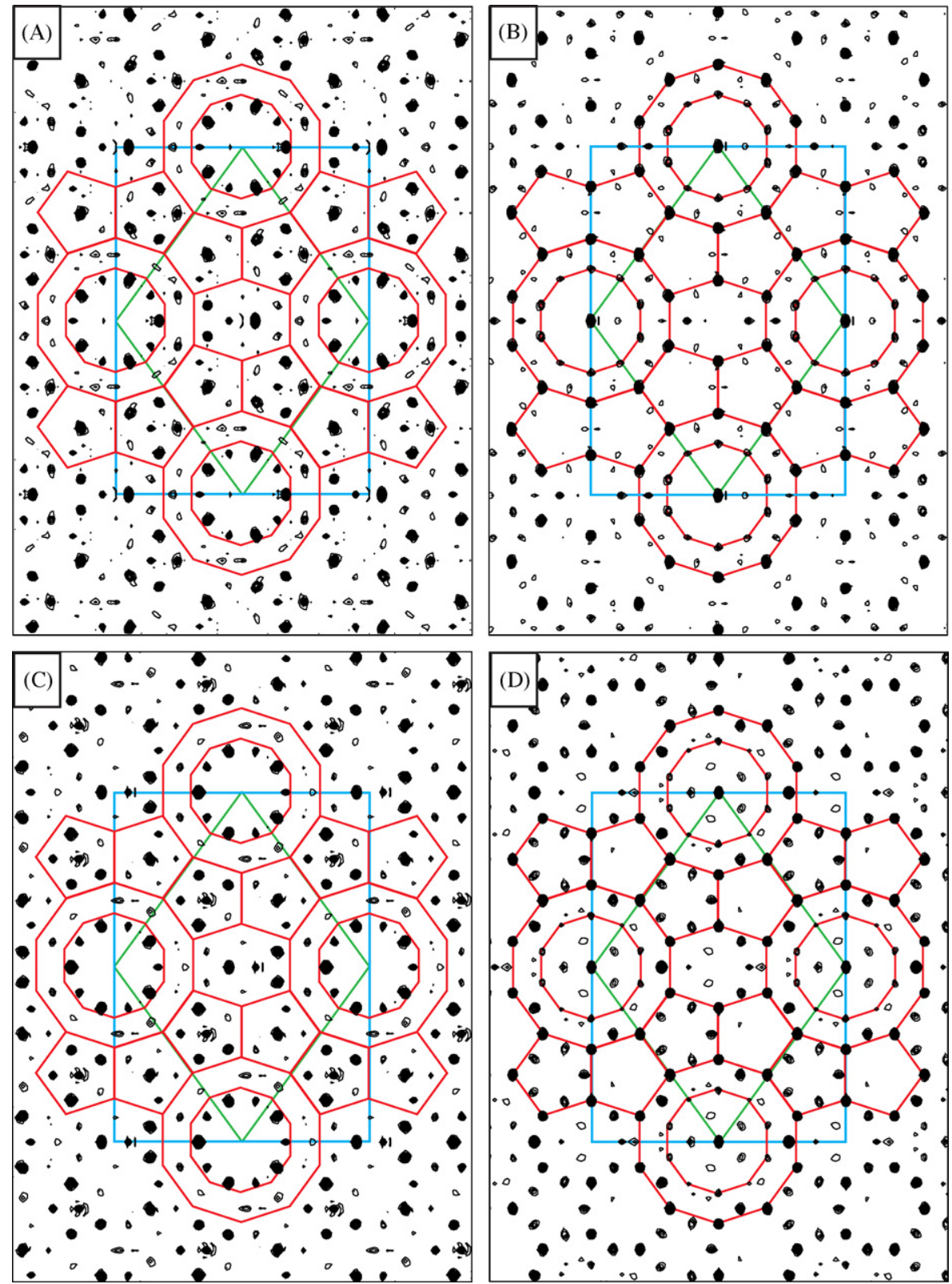

Fig. 10. Electron density maps of the hypothetical $3 / 5$-approximant $(-0.4 \leq x \leq 1.4,-0.4 \leq y \leq 1.4, z)$. The maps are bounded projections with A: $0.002 \leq z \leq 0.086$, B: $0.148 \leq z \leq 0.206$, C: $0.263 \leq z \leq 0.320$, D: $0.400 \leq z \leq 0.430$. The unit cell is marked as well as a Penrose rhomb with edge length of $20.331 \AA$. Characteristic structure motifs, pentagons with edge length of $7.765 \AA$ and decagons are outlined. 
ability of charge flipping for reconstructing electron densities for IMS, immediately suggested that the same method could be successful in the solution of quasicrystal structures with hyperatoms occupying discrete volumes in five- or six-dimensional superspace.

The algorithm has been described in detail by Refs. [22,23] and its generalization towards superspace by Ref. [24]. Here we give only a short account of the basic principle. The structure solution by charge flipping proceeds in iteration cycles. The iteration is initialized by assigning random phases to the experimentally obtained amplitudes of structure factors. From this trial solution, an electron density distribution function is calculated by inverse Fourier transform on a discrete grid of points. Then all grid points with densities below a certain positive threshold $\delta$ are multiplied by -1 (flipped), and new temporary structure factors are calculated by Fourier transform of this modified density. The phases of these temporary structure factors are combined with the observed amplitudes and such a new set of structure factors enters the next iteration cycle. This procedure is repeated until a stable solution is found. Charge flipping always reconstructs the density in the full unit cell without taking the symmetry of the structure into account. The position of the symmetry elements must be therefore located in the reconstructed density map.

The structure of d-Al-Ir-Os was solved by a computer program SUPERFLIP [25] that is designed for the application of the charge-flipping method to structure solution in arbitrary dimensions. All 6782 unique reflections of the data set taken on Xcalibur PX were used while for the pseudo-approximant method only use was made of the subset of 5191 reflections with $I>4 \sigma(I)$. The electron density of d-Al-Ir-Os was reconstructed on a $5 \mathrm{D}$ grid of $24 \times 24 \times 24 \times 24 \times 64$ points. The optimal value of $\delta$ was found automatically by the program. The iteration converged after less than 100 cycles. The electron density was averaged according to the non-centrosymmetric 5D space group $P 10_{5} m c$ since the use of the centrosymmetric space group leads to some unphysically short distances.

The density map obtained by this procedure contained the structural information about the heavy atoms, but the electron density at positions occupied by the light atoms was close to the noise level. To suppress the noise and amplify the weak structural features, the calculations were repeated many times and their results were averaged. The final results shown in Figs. 11-13 were obtained by summing up 175 individual maps. For an explanation of the terminology of the higher-dimensional approach used see Ref. [18] or, for a practical example, [26].

The (1 $\left.\begin{array}{llll}0 & 1 & 1 & 0\end{array}\right)$ section through the 5D unit cell of d-Al-Ir-Os embedded in 5D space shows the positions of all atomic surfaces (hyperatoms) in the unit cell (Fig. 12). This section is very useful in order to identify the atomic layers. The hyperatoms can be grouped into four atomic layers per asymmetric unit. Layers B and $\mathrm{D}$ are almost flat while $\mathrm{A}$ and $\mathrm{C}$ are puckered (up to $\pm 0.5 \AA$ ).

The assignment of letters A-D used in Figs. 10-12 corresponds to each other. On a first glance, the clusters decorating the vertices of the supertiles, i.e. the Penrose rhomb in Fig. 10 and the pentagons in Fig. 12, look very similar. This is not the case, however, although the unit cell of the 3/5-approximant $\left(a_{1}^{\text {app }}=23.901 \AA, a_{2}^{\text {app }}=32.896 \AA\right)$ is large enough to contain

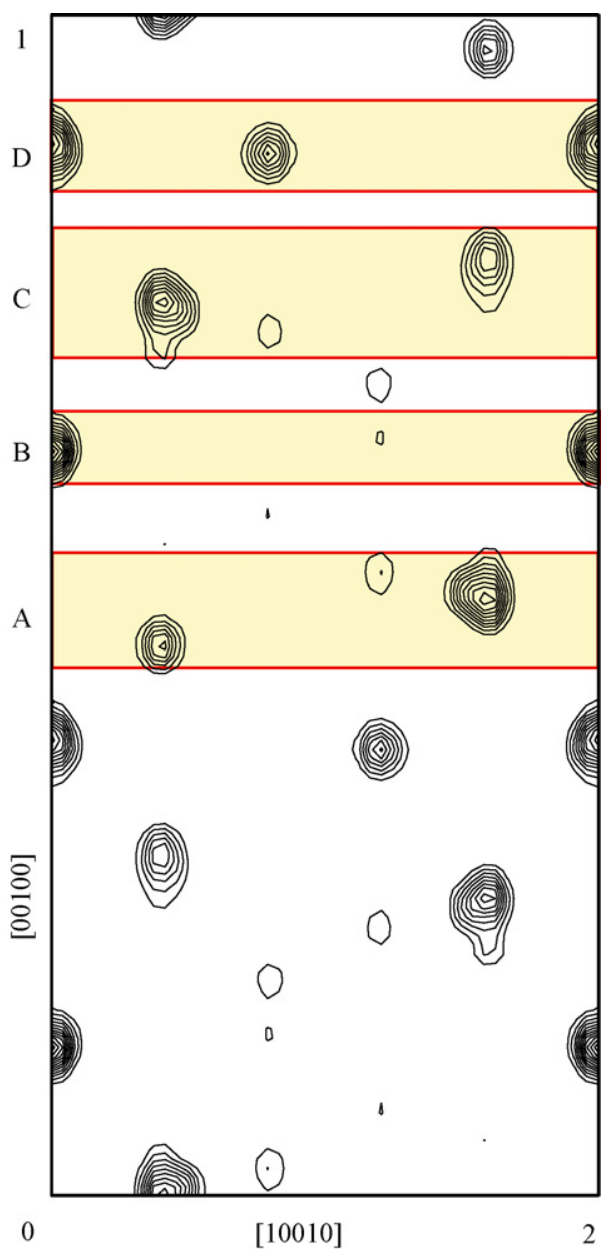

Fig. 11. Special ( $\left.\begin{array}{l}1 \\ 0\end{array} 1110\right)$ section through the 5D unit cell of d-Al-Ir-Os embedded in 5D space. All atomic surfaces (or hyperatoms, for an explanation see [18]) of one 5D unit cell are centered in this section at the sites $(2 i / 50 z 2 i / 50)$, $i=0-4$. The shaded rectangles lettered A-D mark the boundaries of the bounded projections used to produce the corresponding maps A-D depicted in Fig. 12. The numbers at the coordinate axes are in units of $a=1 / a^{*}$. The period in the $\left[\begin{array}{lllll}1 & 0 & 0 & 1 & 0\end{array}\right]$ direction is $2 a$.

a full cluster with $20.150 \AA$ diameter (see Fig. 12). Since the cluster decorates a pentagon tiling with two different pentagon tiles, $\alpha, \beta$, in the quasiperiodic structure, it cannot be properly reproduced by our 3/5-approximant. Due to the $C$-centering, the unit cell contains just one Penrose rhomb, and not a pentagon tile, with edge length $20.331 \AA$. This applies some symmetry constraints to the cluster leading to a partially averaged cluster.

The ordering of the clusters can be derived from larger sections. In Fig. 13 is shown a $193 \AA \times 193 \AA$ projected electron density map. The dominating tile shapes are pentagons, squashed hexagons, boats and skinny rhombs, all together forming decagons. The tiling corresponds to the DT6 tiling shown In Fig. 8 of [27]. In the projection, the decoration of the pentagons appears uniform contrary to what can be seen in the atomic layers (Fig. 12). Particularly in layers A and D of Fig. 12, it can be clearly seen that the decoration of neighboring blue pentagons, $\alpha, \beta$, is different. Due to the action of the $c$-glide planes, $\alpha$ is overlaid with $\beta$. Consequently, this kind of superorder could not be observed by electron microscopic methods. 

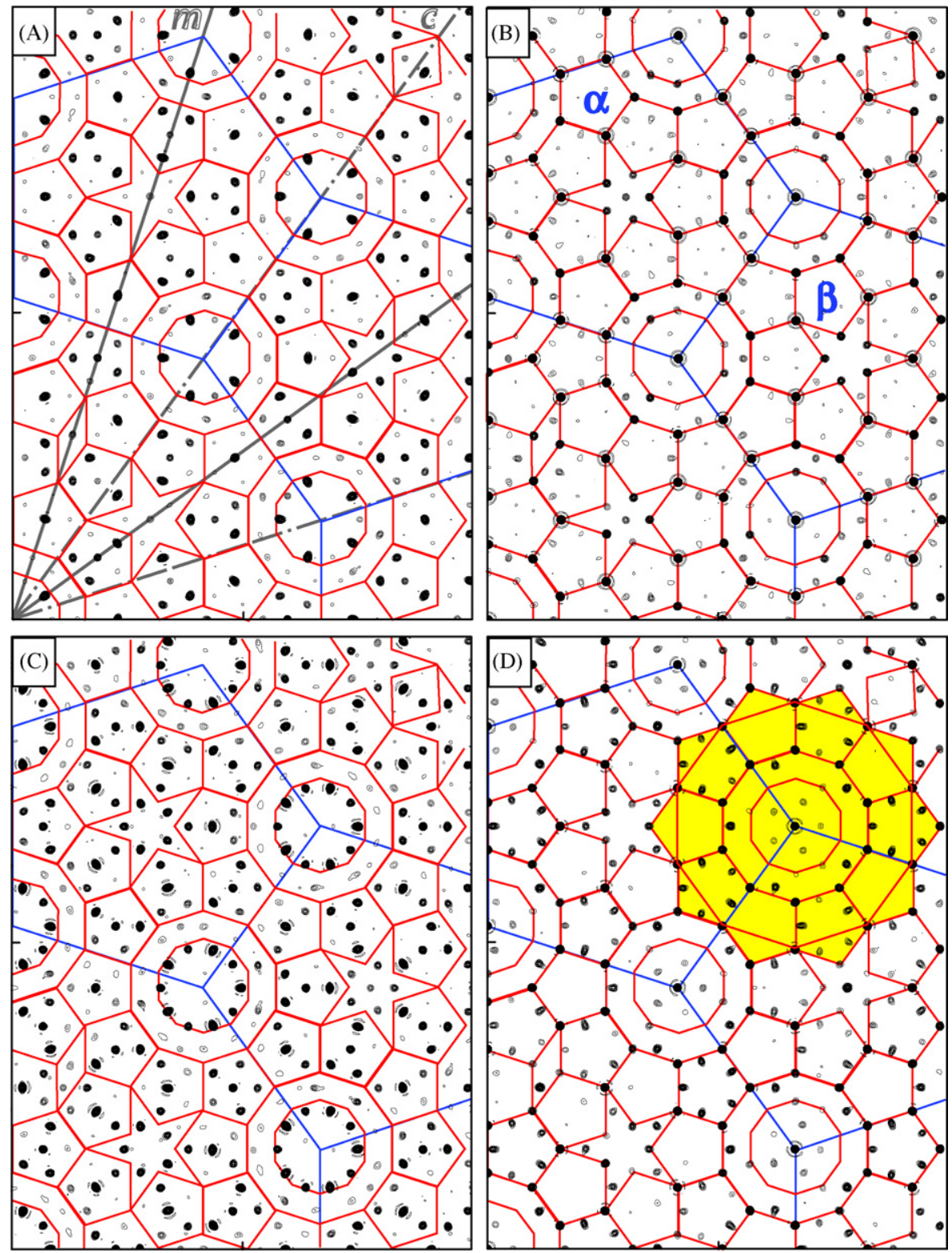

Fig. 12. Bounded projections $(12 a \times 16 a)$ of the electron density of the four symmetrically independent atomic layers, A-D of d-Al-Ir-Os. The lettering refers to the projection boundaries marked in Fig. 11. Large pentagonal tiles with edge length $20.150 \AA$ and characteristic structure motifs, small pentagons (with edge length of $7.697 \AA$ ) and decagons are marked. It can be clearly seen in layers A and D that the decoration of neighboring large pentagons, $\alpha, \beta$, differs significantly.

\subsection{Conclusions}

A new decagonal quasicrystal was found with nominal composition $\sim \mathrm{Al}_{73} \operatorname{Ir}_{14.5} \mathrm{Os}_{12.5}$. It is the quasicrystal with the highest melting temperature, $T_{\mathrm{m}}=1283^{\circ} \mathrm{C}$, known. Its structure is almost isostructural to d-Al-Os-Pd and d-Al-Ni-Ru. The characteristic cluster has $20.150 \AA$ diameter and decorates a pentagonal tiling of that edge length. d-Al-Ir-Os can be seen as Os-stabilized metastable d-Al-Ir. The origin of this stabilization is mainly a slight adjustment of the electron concentration in order to get a better Fermi-surface/Brillouin-zone nesting.

For the determination of its structure two new methods have been applied. The pseudo-approximant method reduces the structure solution problem to a conventional 3D one. A reasonable model of the quasicrystal-forming cluster can only be obtained if the unit cell of the approximant is large enough. In the present case, the 4/6-approximant would have been more appropriate to give the full picture. The 'charge-flipping' method proved to be very successful in reconstructing the phases of the structure factors. In all cases, in which it does not make sense to perform a very time-consuming full higher-dimensional structure analysis (atomic surface modeling), the 'charge-flipping' method can be the method of choice. The quality of the electron density maps can be even improved by applying the maximumentropy method. This requires however the determination of $F(00000)$. One possibility to obtain the structure factor in the 


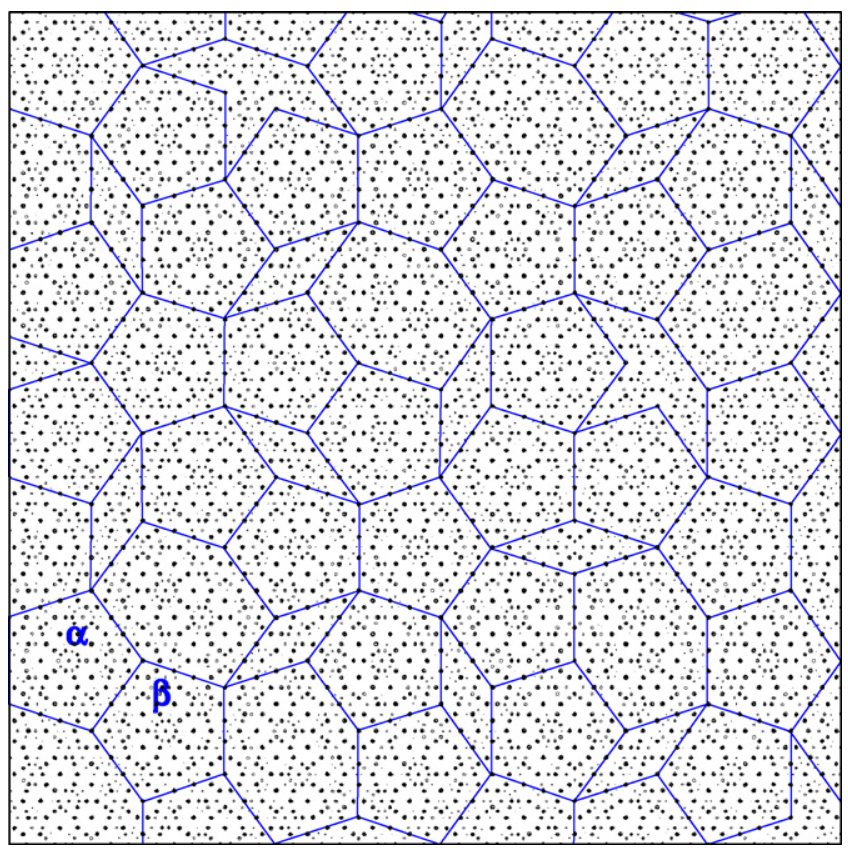

Fig. 13. $193 \AA \times 193 \AA$ projected electron density map. The cluster centers are connected by lines to mark characteristic tile shapes (decagons, pentagons, squashed hexagons, skinny rhombs). In the projected structure, the pentagon tiles $\alpha$ and $\beta$ appear the same. The edge length of the tiles amounts to $20.150 \AA$. The tiling resembles the DT6 tiling [27].

origin of the reciprocal space could be the pseudo-approximant method.

\section{Acknowledgement}

Financial support under grant SNF 200020-105158 is gratefully acknowledged.

\section{References}

[1] P.J. Hill, L.A. Cornish, M.J. Witcomb, J. Alloys Compd. 291 (1999) $130-144$.
[2] H. Okamoto, Desk Handbook. Phase Diagrams for Binary Alloys, ASM, Materials Park, 2000.

[3] M. Boström, R. Niewa, Y. Prots, Y. Grin, J. Solid-State Chem. 178 (2005) 339-345.

[4] S. Katrych, V. Gramlich, W. Steurer, J. Alloys Compd. 407 (2006) 132140.

[5] S. Mi, S. Balanetskyy, B. Grushko, Intermetallics 11 (2003) 643-649.

[6] Z.M. Wang, Y.Q. Gao, K.H. Kuo, J. Less-Common Met. 163 (1990) $19-26$.

[7] R. Wang, L. Ma, K.H. Kuo, J. Less-Common Met. 163 (1990) 27-35.

[8] Yu. Grin, K. Peters, U. Burkhardt, K. Gotzmann, M. Ellner, Z. Kristallogr. 212 (1997) 439-444.

[9] P. Villars, L.D. Calvert, Pearson Handbook of Crystallographic Data for Intermetallic Phases, 2nd ed., Ohio, ASM International, Materials Park, 1991, 4 volumes.

[10] L.E. Edshammar, Acta Chem. Scand. 18 (1964) 2294-2302.

[11] R. Ferro, R. Capelli, R. Marazza, S. Delfino, Atti Accad. Naz. Lincei, Cl. Fis Mat. Nat. Rendu. 45 (1968) 556.

[12] A.P. Tsai, A. Inoue, T. Masumoto, Philos. Magn. Lett. 64 (1991) 163 167.

[13] W. Steurer, F. Frey, Phase Trans. 67 (1998) 319-361.

[14] B. Zhang, X. Li, W. Steurer, J. Schneider, F. Frey, Philos. Magn. Lett. 72 (1995) 239-244.

[15] W. Steurer, Z. Kristallogr. 219 (2004) 391-446.

[16] A. Cervellino, Higher-dimensional modelling of decagonal quasicrystal structures. ETH Zurich Thesis 14023, 2000.

[17] T. Scholpp, Dekagonale Quasikristalle und Approximanten in den Systemen Al-Ni-Co, Al-Cu-Co und Al-Ni-Ru. Diss. ETH Nr. 14292, 2001.

[18] W. Steurer, T. Haibach, in: Z.M. Stadnik (Ed.), Physical Properties of Quasicrystals, Springer-Verlag, Berlin, 1999.

[19] G. Sheldrick, SHELXS-97. Program for the Solution of Crystal Structures, University of Göttingen, Germany, 1997.

[20] G. Sheldrick, SHELXL-97. Program for the Refinement of Crystal Structures, University of Göttingen, Germany, 1997.

[21] W. Sun, T. Ohsuna, K. Hiraga, Philos. Magn. Lett. 81 (2001) 195-425.

[22] G. Oszlányi, A. Süto, Acta. Crystallogr. A60 (2004) 134-141.

[23] G. Oszlányi, A. Süto, Acta. Crystallogr. A61 (2005) 147-152.

[24] L. Palatinus, Acta Crystallogr. A60 (2004) 604-610.

[25] SUPERFLIP-a computer program for application of the charge flipping algorithm in superspace of arbitrary dimension. Available on-line at: http://superspace.epfl.ch/superflip.

[26] W. Steurer, T. Haibach, B. Zhang, S. Kek, R. Lück, Acta Crystallogr. B 49 (1993) 661-675.

[27] Z. Masakova, J. Patera, J. Zich, J. Phys. A 38 (2005) 1947-1960. 